\title{
Utilidad de los modelos clínicos en la predicción de tromboembolia pulmonar
}

\author{
J. L. ALONSO MARTÍNEZ, J. L. GARCÍA SANCHOTENA', M. L. ABÍNZANO \\ GUILLÉN, M. A. URBIETA ECHEZARRETA, F. J. ANNICCHERICO SÁNCHEZ, \\ V. FERNÁNDEZ LADRÓN
}

Servicios de Medicina Interna y ${ }^{\prime}$ Radiología. Hospital de Navarra. Pamplona

UTILITY OF THE CLINICAL MODELS FOR PREDICTING PULMONARY THROMBOEMBOLISM

\begin{abstract}
RESUMEN
Objetivo: Valorar la efectividad de los modelos de predicción clínica de tromboembolia pulmonar (TEP).

Material y métodos: Aplicación retrospectiva de los tres modelos (canadiense, Ginebra y Pisa) a pacientes diagnosticados inequívocamente de TEP por arteriografía o tomografía computerizada (TAC) helicoidal.

Resultados: Analizamos 120 pacientes [edad media $71 \pm 13$ años, varones $63(52 \%)$ ] diagnosticados $19(16 \%)$ por arteriografía y 101 $(84 \%)$ por TAC helicoidal. La distribución en el modelo canadiense fue alta probabilidad $24 \%$, intermedia $59 \%$ y baja $17 \%$. En el modelo Ginebra fue de alta probabilidad $21 \%$, intermedia $69 \%$ y baja $10 \%$. En el modelo Pisa la distribución fue de alta probabilidad 49\%, intermedia $45 \%$ y baja $6 \%$. La sensibilidad de los modelos fue respectivamente 0,59 , 0,67 y 0,89

Factores que condicionaron baja probabilidad fueron en el modelo canadiense la frecuencia cardiaca, la ausencia de signos de trombosis venosa, la existencia de un diagnóstico alternativo y la baja tasa de cáncer. En el modelo Ginebra fueron la edad, la frecuencia cardiaca y la $\mathrm{PaO}_{2}$. En el modelo Pisa condicionaron baja probabilidad diagnóstica la radiografía de tórax normal y la existencia de edema pulmonar.

Conclusiones: En los tres modelos de predicción clínica de TEP se observan deficiencias, siendo el modelo Pisa el más adecuado para predecir la probabilidad de TEP. La probabilidad intermedia en modo alguno debe servir para excluir el diagnóstico, y aún con baja probabilidad un número de pacientes en los tres modelos tendrán TEP. En conjunto la utilidad de los modelos de predicción clínica muestran un valor limitado.
\end{abstract}

PALABRAS CLAVE: Tromboembolia pulmonar. Tromboembolismo pulmonar. Modelos clínicos. Predicción clínica.

\section{ABSTRACT}

Objective: We considered to evaluate the efectivity of the clinical models for predicting pulmonary thromboembolism (PE).

Methods: Retrospective application of three published clinical models (Wells or Canadian model, Geneva model and Pisa model) to patients unequivocally diagnosed of acute PE.

Results: We evaluate 120 patients [Mean age $71 \pm 13$ years, males 63 $(52 \%)$ ]: Nineteen $(16 \%)$ diagnosed with pulmonary arteriography and $101(84 \%)$ diagnosed with helical computed tomography.

In the Canadian model $24 \%$ patients were of high clinical probability, 59\% intermediate and $17 \%$ low clinical probability. In Geneva model $21 \%$ patients belonged to high clinical probability, $69 \%$ intermediate and $10 \%$ low clinical probability. In Pisa model $49 \%$ patients were of high clinical probability, $45 \%$ intermediate and $6 \%$ of low clinical probability. Sensitivity was $0.59,0.67$ and 0.89 respectively.

Factors associated with low probability were in Canadian model the heart rate, the absence of signs of deep venous thrombosis, the presence of an alternative diagnosis and the low rate of cancer. In Geneva model, age, normal heart rate and $\mathrm{PaO}$, higher $70 \mathrm{~mm} \mathrm{Hg}$ were associated with low probability, while in Pisa model normal chest $X$-Ray and radiological signs of pulmonary oedema were also associated with low clinical probability.

Conclusions: Although all three clinical model showed deficiencies Pisa model was the most suitable clinical model for predicting PE. An intermediate clinical probability in the three models, should not serve to rule out PE, besides it is remarkable that patients with low clinical probability still could have PE, providing for clinical models with a limited value.

KEY WORDS: Pulmonary thromboembolism. Clinical models. Prediction of pulmonary thromboembolism.

Alonso Martínez JL, García Sanchotena JL, Abínzano Guillén ML, Urbieta Echezarreta MA, Anniccherico Sánchez. FJ, Fernández Ladrón V. Utilidad de los modelos clínicos en la predicción de tromboembolia pulmonar. An Med Interna (Madrid) 2006; 23: 264-268.

\section{INTRODUCCIÓN}

La enfermedad tromboembólica venosa (ETEV), y su manifestación de mayor gravedad el tromboembolismo pulmonar (TEP) es un proceso prevalente (1-3) y con frecuencia grave (4). Las estrategias diagnósticas del TEP han ido cambiando desde el aserto de que es necesario un alto índice de sospecha clínica, debido a la inespecificidad de sus manifestaciones clínicas y la fácil confusión con otros procesos cardiovasculares y respiratorios, al establecimiento de la probabili-

Trabajo aceptado: 13 de enero de 2006 
dad clínica antes de la utilización de pruebas diagnósticas (59).

Estas pruebas diagnósticas también han ido modificándose en el tiempo con la rápida introducción de la tomografía axial computerizada (TAC) helicoidal y la aceptación como prueba de imagen diagnóstica principal en el TEP (10-14).

Aunque se han publicado en nuestro país $(15,16)$ y en otros $(17,18)$, la utilidad de diferentes modelos clínicos de predicción de probabilidad sobre pacientes ya diagnosticados de TEP, estos han sido basados en diagnósticos principalmente realizados con gammagrafia pulmonar de ventilación/perfusión.

Nuestro objetivo fue saber la utilidad de los modelos de predicción clínica en una serie de pacientes diagnosticados inequívocamente de TEP.

\section{MATERIAL Y MÉTODOS}

Para ver la utilidad de los tres modelos de probabilidad clínica de tromboembolismo pulmonar publicados (Tabla I) y saber, por una parte si existen diferencias entre ellos, y por otra parte cual es le mejor método en nuestros pacientes, aplicamos retrospectivamente los tres modelos a 120 pacientes consecutivos diagnosticados inequívocamente de tromboembolismo pulmonar por arteriografía pulmonar (Angiografo Siemens Angiostar, Alemania) o TAC helicoidal (General Electric Medical Systems EE.UU: desde mayo de 1992 a mayo del 2005.
Cada ítem fue recogido de las historias clínicas y de los informes de alta estructurados en los que constan edad, sexo, antecedentes clínicos, historia clínica actual, exploración física, gasometría arterial y datos de análisis habituales, descripción de las radiografías de tórax, descripción del electrocardiograma, descripción de la arteriografía pulmonar y de la TAC helicoidal y el diagnóstico de alta del paciente.

Consideramos incertidumbre diagnóstica en el modelo Wells cuando existían antecedentes de enfermedad cardiovascular o respiratoria crónica previa que dificultaba la distinción clínica, y también cuando el diagnóstico de ingreso dado por los médicos del servicio de urgencias era otra enfermedad cardiovascular o respiratoria, asignándole entonces la categoría de otro diagnóstico más probable. Consideramos para todos los modelos inmovilización, cuando existía trastorno de la movilización o ingreso hospitalario en las cuatro semanas previas. Consideramos el epígrafe cirugía previa cuando se realizó cirugía en las cuatro semanas previas. Consideramos la presencia de hemoptisis tanto al esputo hemoptóico como a la hemoptisis franca. Consideramos la presencia de cáncer en aquellos pacientes con diagnóstico en el último año, en tratamiento activo o en tratamiento paliativo.

En el modelo Pisa como historia de tromboflebitis interpretamos además de los signos clínicos de dolor venoso y edema, la existencia en las 4 semanas recientes de dolor y/o edema en una pierna. En el apartado disnea súbita se incluyó tanto la disnea de aparición súbita como el cambio de la disnea habitual cuando existía un proceso de base potencialmente causante de disnea.

TABLA I

MODELOS CLÍNICOS DE PREDICCIÓN DE PROBABILIDAD DE TROMBOEMBOLISMO PULMONAR

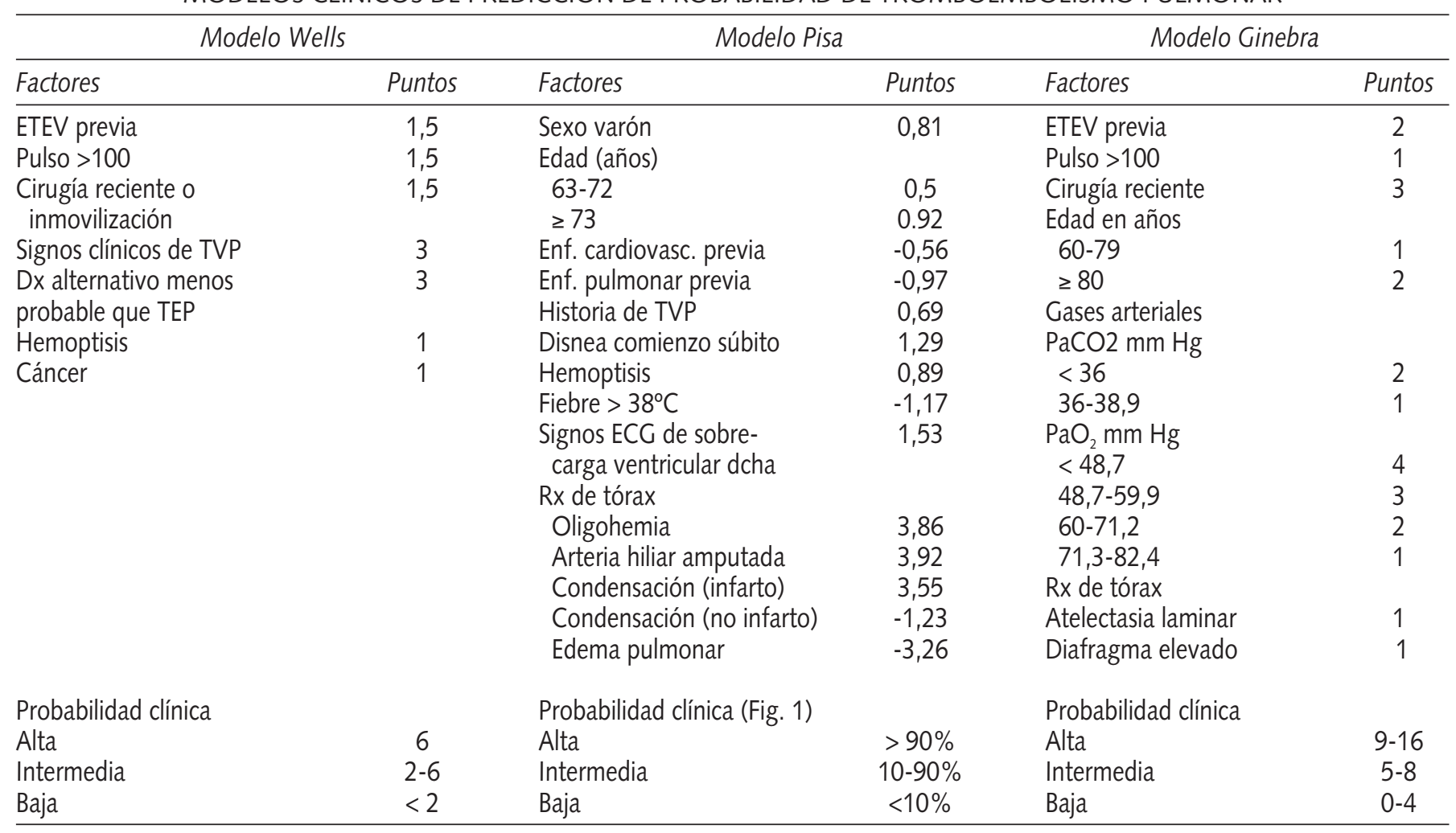

ETEV: enfermedad tromboembólica venosa; TVP: trombosis venosa profunda; Dx: diagnóstico. 


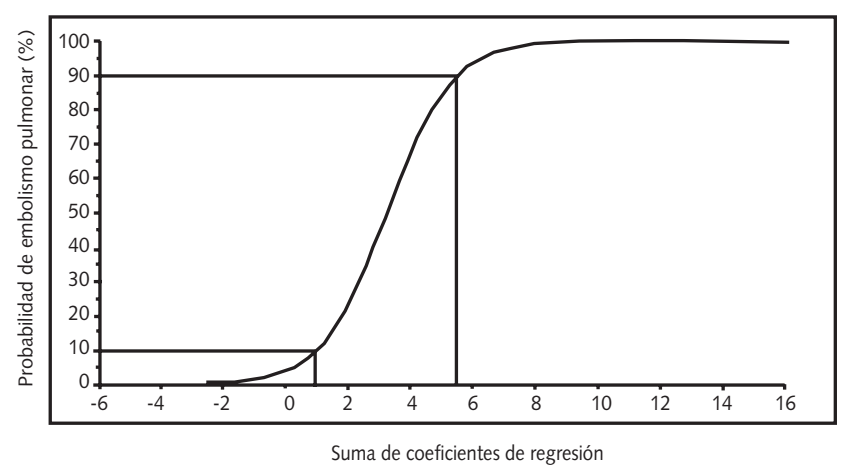

Fig. 1. Para estimar la probabilidad de embolismo pulmonar hacer la suma algebraica de todos los coeficientes y aplicarlos en el eje de la X. Después leer la probabilidad correspondiente en el eje de la Y.

Los valores gasométricos utilizados fueron los de la gasometría arterial realizada al ingreso del paciente.

\section{ANÁLISIS ESTADÍSTICO}

Las variables continuas se expresan en medias \pm la desviación estándar. Para comparación de variables continuas se utilizó la prueba de la t de Student cuando tenían distribución normal y la prueba de la U de Mann Whitney cuando tenían distribución no paramétrica. Para comparación de variables continuas utilizamos ANOVA en las variables de distribución normal y la prueba de Kruskal Wallis cuando no mostraban distribución normal.

Las variables cualitativas se expresan en porcentajes. Para su análisis se ha utilizado la prueba de la comparación de pro- porciones y la prueba exacta de Fisher de dos colas cuando el número era menor de 5. Calculamos la sensibilidad, con la expresión $\mathrm{a} / \mathrm{a}+\mathrm{c}$, donde a son los pacientes de alta probabilidad y c los de baja probabilidad.

Se consideró significación estadística un valor de $\mathrm{p}<0,05$.

\section{RESULTADOS}

Analizamos 120 pacientes diagnosticados de tromboembolisno pulmonar de forma inequívoca aplicando los tres modelos descritos de probabilidad pretest de TEP.

La edad media de los pacientes fue de $71 \pm 13$ años. La edad media de las mujeres fue de $75 \pm 12$ años y la de los varones de $68 \pm 13$ años $(\mathrm{p}<0,01)$. Varones $63(52 \%)$

El diagnóstico se realizó por arteriografía pulmonar en 19 (16\%) pacientes y por TAC helicoidal en 101 (84\%) pacientes.

La tabla II recoge la ocurrencia de cada ítem en cada modelo de predicción clínica de TEP. La tabla III muestra los valores absolutos y las proporciones de las probabilidades de los tres modelos.

La sensibilidad del modelo Wells fue de 0,59 , la del modelo Ginebra 0,67 y la del modelo Pisa 0,89. Debido a que todos los pacientes son enfermos de tromboembolismo pulmonar, no se pudo calcular la especificidad ni los valores predictivos.

En la comparación de los tres modelos, en los pacientes con alta probabilidad el modelo Pisa fue superior al de Wells ( $\mathrm{p}=0,004$, IC al 95\% 0,12 a 0,37), y al modelo Ginebra $(\mathrm{p}=0,0014$, IC al $95 \% 0,15$ a 0,40$)$.

La proporción de probabilidad intermedia fue menor en el modelo Pisa que en el modelo Ginebra ( $p=0,03$ IC al 95\% 0,11 a 0,37 ) no habiendo diferencias con el modelo Wells.

Hubo menos pacientes con probabilidad baja en el modelo Pisa respecto al modelo Wells ( $\mathrm{p}<0,02$, IC al 95\% de 0,02 a

\section{TABLA ॥}

\section{OCURRENCIA DE EVENTOS EN CADA MODELO}

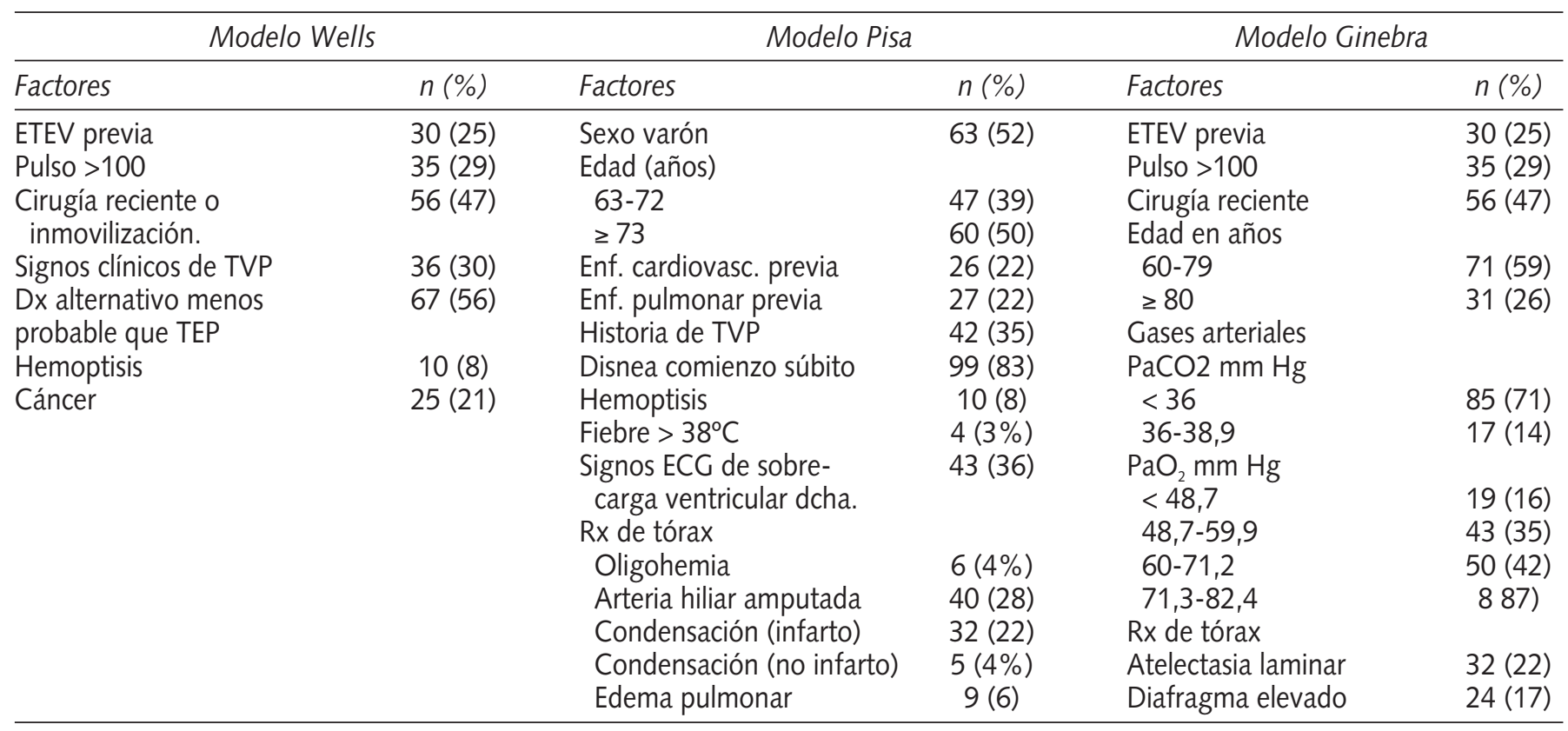

ETEV: enfermedad tromboembólica venosa; TVP: trombosis venosa profunda; Dx: diagnóstico. 
TABLA III

DISTRIBUCIÓN DE PROBABILIDADES EN CADA MODELO

\begin{tabular}{lccc}
\hline Probabilidad & Wells & Ginebra & Pisa \\
\hline Alta & $29(24 \%)$ & $25(21 \%)$ & $59(49 \%)$ \\
Media & $71(59 \%)$ & $83(69 \%)$ & $54(45 \%)$ \\
Baja & $20(17 \%)$ & $12(10 \%)$ & $7(6 \%)$ \\
\hline
\end{tabular}

0,20), no habiendo diferencias con respecto al modelo Ginebra.

La tabla IV refleja los factores que en cada modelo determinaron una baja probabilidad comparado con alta probabilidad, o dicho de otra manera resultados que hubieran propiciado que los pacientes se hubieran escapado al diagnóstico.

\section{TABLA IV}

COMPARACIÓN DE FACTORES DIFERENCIALES ENTRE ALTA Y BAJA PROBABILIDAD CLIINICA

\begin{tabular}{lllc}
\hline Wells & Alta probabilidad & Baja probabilidad & $p$ \\
\hline Frecuencia Pulso & $90,51 \pm 16,46$ & $80,21 \pm 17,12$ & $<, 05$ \\
No Signos de TVP & $24 \%$ & $95 \%$ IC 95\% 0,48-0,93 & $<, 001$ \\
Existe Dx. alternativo & $7 \%$ & $95 \%$ IC 95\% 0,70-1,05 & $<, 001$ \\
Cáncer & $34 \%$ & $5 \%$ IC $95 \% 0,05-0,53$ & $<, 05$ \\
\hline Cinebra & & & \\
\hline Edad & $75 \pm 11$ & $62 \pm 12$ & $<, 05$ \\
Frecuencia Pulso & $94,2 \pm 17,04$ & $76,3 \pm 17,7$ & $<, 05$ \\
PaO $_{\text {(mm Hg) }}$ & $53,44 \pm 8,99$ & $73,25 \pm 10,59$ & $<, 01$ \\
\hline Pisa & & & \\
\hline Rx tórax normal & $3 \%$ & $57 \%$ IC 95\% 0,08-0,98 & $<, 005$ \\
Edema pulmón Rx & $0 \%$ & $43 \%$ IC 95\% 0,17-0,87 & $<, 005$ \\
\hline
\end{tabular}

\section{DISCUSIÓN}

La alta tasa de mortalidad del TEP y la inespecificidad de los síntomas han producido simultáneamente una sobreindicación de pruebas diagnósticas y una alta tasa de infradiagnóstico, para intentar solucionar esta situación se han generado modelos de predicción clínica de TEP (5-9).

La probabilidad clínica de padecer una tromboembolia pulmonar se evalúa fundamentalmente con alguno de los tres modelos de predicción publicados, el modelo Ginebra, el modelo Pisa y el modelo Wells o modelo canadiense, y aunque han sido diseñados como predicción pre-test, nos pareció de utilidad saber la precisión que ofrecen en pacientes ya diagnosticados de tromboembolia pulmonar por un método de imagen que de un diagnostico inequívoco de TEP, diferente de la gammagrafia pulmonar.

La gammagrafía pulmonar aunque es útil, supone un método indirecto de diagnóstico, ya que no detecta el émbolo en sí, sino sus consecuencias, y muchos otros problemas clínicos pueden producir defectos de perfusión (neumonías, bullas, derrame pleural, etc), y cuando el paciente tiene un infarto pulmonar la ventilación en la gammagrafía también puede ofrecer alteración emparejada con la perfusión reduciéndose la probabilidad diagnostica. Si la gammagrafía es de alta probabilidad, $85 \%$ de los pacientes tendrán una tromboembolia pulmonar, lo que significa que $15 \%$ de los pacientes serán tratados innecesariamente con anticoagulantes. Pero solo una minoría de pacientes con verdadero TEP tendrán una gammagrafía de alta probabilidad, siendo en conjunto la sensibilidad del $41 \%$ y la especificidad del $97 \%(19,20)$. Todo esto, en nuestra opinión, introduce un sesgo en la valoración de los modelos de predicción clínica pretest cuando se basan en diagnóstico gammagráfico, por ello elegimos pacientes en los que se demostraron trombos en las arterias pulmonares, bien por arteriografía o bien por TAC helicoidal.

Del análisis de nuestros resultados se desprende que los tres modelos no son equiparables, y que el método que mejor se adapta al diagnostico de TEP es el modelo Pisa, con una mayor proporción de pacientes de alta probabilidad en comparación con los otros dos modelos.

En los tres modelos, una gran parte de pacientes se encuadra dentro de la probabilidad intermedia, lo que significa en el terreno de la practica clínica, que una probabilidad intermedia aún tiene una proporción de diagnostico positivos entre el 55 y el $80 \%$, por lo que de ningún modo debe usarse para descartar la tromboembolia pulmonar con ninguno de los tres modelos.

Por otra parte una probabilidad baja con los tres modelos, aun mantiene una proporción de diagnósticos positivos de TEP no desdeñable teniendo en cuenta la potencial gravedad de la omisión del diagnóstico. El modelo Pisa es el que tiene una proporción más pequeña de TEP en la categoría de baja probabilidad.

Los factores que propiciaron que los tres modelos dieran como resultado una baja probabilidad son diferentes en los tres modelos. La debilidad del modelo canadiense se concreta por una parte en la frecuencia cardiaca, de modo que una frecuencia cardiaca normal no permite descartar un TEP, dependiendo ésta de la reserva cardiovascular del paciente, de la toma de fármacos como tratamiento habitual que causen bradicardia y de la superficie circulatoria pulmonar ocluida, y por otra parte de la ausencia de signos de TVP, ya que solo la tercera parte de los pacientes con tromboembolia pulmonar tienen signos de TVP. Otro punto que ofrece debilidad al modelo canadiense es la existencia de un diagnóstico alternativo más o menos probable que el TEP. En este apartado es preciso tener en cuenta varios factores: la experiencia del médico, la inespecificidad de la clínica que puede similar múltiples procesos cardiovasculares y respiratorios y los deseos de diagnóstico positivo de los profesionales para hacer casuística. Parece que la experiencia de los médicos evaluadores aumenta la probabilidad pre-test de diagnóstico de TEP, aunque sigue sin estar claro si es mejor utilizar alguno de los modelos o fiarse del hacer clínico habitual (21).

El modelo Ginebra tiene la debilidad, en nuestra opinión, de basarse en exceso en la gasometría arterial, cuando está demostrado que tanto una gasometría arterial como un gradiente alveolo arterial para el oxígeno normales no descartan apropiadamente un TEP $(22,23)$.

El modelo Pisa tiene la dificultad de ser más engorroso en el cálculo, precisando calculadora y transportar el resultado a una curva de probabilidad. Se basa de forma importante en datos radiológicos que pueden ser difíciles de interpretar y 
estar sometido a variabilidad interobservador, necesitándose experiencia para su evaluación.

La limitación de este estudio radica fundamentalmente en que los médicos no evaluaron directamente a los pacientes sino que se estudiaron los datos clínicos, analíticos y radioló- gicos de forma indirecta y retrospectiva, por lo que debe ser catalogado como una simulación clínica no como una evaluación clínica real.

En conjunto nuestros datos favorecen al modelo Pisa de evaluación de probabilidad clínica de padecer un TEP.

\section{Bibliografía}

1. Goldhaber SZ. Thrombolysis for pulmonary embolism. Prog Cardiovasc Dis 1991; 34: 113-4

2. Stein PD, Huang H, Ofzal A, Noor HA. Incidence of acute pulmonary embolism in a general hospital. Relation to age, sex and race. Chest 1999; 116: 909-15

3. Alonso Martínez JL, Echegaray Agara M, Urbieta Echezarreta MA, Abínzano Guillén ML, García Sanchotena JL, González Arencibia C. Embolismo pulonar agudo. Un registro de 10 años: de mayo d 1992 a mayo de 2002. Rev Clin Esp 2004; 204: 521-7

4. Echegaray Agara M, Alonso Martínez JL, Urbieta Echezarreta MA, Abínzano Guillén ML, González Arencibia C, Solano remirez M. Tromboembolismo pulmonar: evolución a largo plazo y epidemiología clínica. An Med Interna (Madrid) 20: 451-6

5. Wells PS, Anderson DR, Rodger M, Ginsberg JS, Kearon C, Gent M, et al. Derivation of a simple model to categorize patients probability of pulmonary embolism: increasing de models utility with the simpliRED D-dimer. Thromb Haemost 2000: 83: 416-20

6. Wells PS, Ginsberg JS, Anderson DR, Kearon C, Gent M, Turpie AG, et al. Use of a clinical model for safe management of patients with suspetd pulmonary embolism. Ann Intern Med 1998; 129: 995-1005

7. Wicki J, Perneger TV, Junod AF, Bounemeaux H, Perrier A. Assessing clinical probability of pulmonary embolism in the emergency ward. A simple score. Arch Intern Med 2001; 161: 92-97

8. Miniati M, Monti S, Bottai M. A Structured clinical model for predicting the probability of pulmonary embolism. Am J Med 2003; 114: 1739

9. Miniati M, Bottai M, Monti S. Comparison of 3 clinocal models for predicting the probability of pulmonary embolism. Medicine 2005; 84: 107-114

10. Perrier A, Roy PM, Sanchez O, Le Gal G, Meyer G, Gourdier AL, et al. Multidetector-row computed tomography in suspected pulonary embolism. N Engl J Med 2005; 352: 1760-8

11. Perrier A, Howarth N, Didier D, Loubeyre P, Unger PF, de Moerloose $\mathrm{P}$, et al. Performance of helical computed tomography in unselected outpatients with suspected acute pulmonary embolism. Ann Intern Med 2001; 135: 88-97

12. Donato AA, Scheirer JJ, Atwell MS, Gramp J, Duszak R. Clinical out-

comes in patients with suspected acute pulmonary embolism and negative helical computed tomography results in whom anticoagulation was withheld Arch Intern Med 2003; 163: 2033-8

13. Moores LK, Jackson Jr WL, Shorr AF, Jackson JL. Meta-analysis: outcomes in patients with suspected pulmonary embolism managed with computed tomographic pulmonary angiography . Ann Intern Med 2004; 141: $866-874$

14. van Strijen MJL, Monye W, Schiereck J, Kieff GJ, Prins MH, Huisman $\mathrm{MV}$, et al. Single-detector helical computed tomography as the primary diagnostic test in suspetd pulmonary embolism: a multicenter clinical management study of 510 patients. Ann Intern Med 2003; 138: 307-14

15. Campo JF, Hernández MJ, Anta M, Saro G, Olmos JM, Riancho JA. Concordancia en el diagnóstico de la tromboembolia pulmonar. Med Clin (Barc) 1988; 111: 121-4

16. Calvo Romero JM, Bureo Dacal P, Pérez Miranda M. Modelos de valoración de la probabilidad clñinica en pacientes con tromboembolia pulmonar. Med Clin (Barc) 2003; 120: 456-7

17. Chagnon I, Bounemeaux H, Aujesky D, Roy PM, Gourdier AL, Cornuz $\mathrm{J}$, et al. Comparison of two cliical predicitions rules and implicit assessment among patients with suspected pulmonary embolism. Am J Med 2002; 113: 269-75

18. Tamariz LJ, Eng J, Segal JB, Krishnan JA, Bolger DT, Streiff MB, et al. Usefulness of clinical prediction rules for the diagnosis of venous thromboembolism: a systematic review. Am J Med 2004; 117: 676-84

19. The PIOPED investigators. Value of the ventilation/perfusion scan in acute pulmonary embolism. JAMA 1990; 263: 2753-9

20. Hull RD, Hirsh J, Carter CJ, Jay RM, Dodd PE, Ockelford PA, et al. Pulmonary angiography, ventilation lung scanning and venography for clinically suspected pulmonary embolism with abnormal perfusion lung scan. Ann Intern Med 1983; 98: 891-9

21. Kabrhel C, Camargo CA, Goldhaber SZ. Clinical gestalt and the diagnosis of pulmonary embolism. Chest 2005; 129: 1627-30

22. Stein PD, Goldhaber SZ, Henry JW, Miller AC. Arterial blood gas analysis in the assessment of suspected acute pulmonary embolism. Chest 1996; 109: 78-81

23. Stein PD, Goldhaber SZ, Henry JW. Alveolar-arterial oxygen gradient in the assessment of acute pulmonary embolism. Chest 1995; 107: 139-43. 